\title{
FIJACION PRIMARIA Y VARIACIONES MORFOLOGICAS, DURANTE LA METAMORFOSIS DE ALGUNOS BIVALVOS CHILENOS
}

\author{
JUAN URIBE BARICHIVITH Y DANIEL LOPEZ STEFONI \\ Universidad de Chile, Sede Osorno, \\ Osorno, Chile
}

\section{SYNOPSIS}

The larval primary settlement and the changes originated during the metamorphosis of some species of Chilean bivalves, are companati. vely sturied, being described, the larval and post - larval stages from the veliconcha to late plantigrade. By observing in ropes "anchovetera" nets and plankton samples, in the mitiliculture of Codihué $\left(41^{\circ} 46^{\prime} \mathrm{S} ; 73^{\circ} 24^{\prime} \mathrm{W}\right)$, it was verified for Mytilus chilensis Hupé, 1854 and Aulacomya ater (Molina, 1782), primary settlement on the filamentous algae of genera Enteromorpha with an average size of $33^{\circ} \mu \mathrm{high}$, minimum average of definitive settlement, byssus positional changes, etc. Information concerning average and number of larval and post -larval attach. ment to different deep and inmersion period and morphological characters of larval and post - larval of: Banikia martensi Stempell, 1898 (Tere. dinidae), Pholas chiloensis (Molina, 1782) (Pholadidae) y Chlamys patriae Doello Jurado, 1918 (Pectinidae), are also given.

\section{Introduccion}

Uno de los mayores problemas en el cultivo de los mitílidos: Mytilus chilensis Hupé, 1854, Choromytilus chorus (Molina, 1782) y Aulacomya ater (Molina, 1782), en el Sur de Chile, lo constituye el escaso control de la captación de juveniles en sustratos artificiales, planteándose la urgencia de investigaciones complementarias al problema, para asegurar una explotación adecuada (Yáñez), 1974). Aun cuando existe información respecto a ciclos de madurez sexual y períodos de desove (Tomicic, 1966; Lozada, 1968; Solis \& Lozada, 1971; Cifuentes, 1975 etc), presencia de huevos y larvas en el plancton (Padilla, 1973) y épocas de fijación de semilla (Yáñez, 1974; López et al., 1975), la identificación larval ha sido posible sólo a nivel de familia (Solis et al., 1976), determinándose también algunas características, en base a la terminología de Carriker (1961) de las fases planctónicas y plantígrados de mitílidos de Isla Teresa (Padilla, 1973). Sobre los factores que influencian el comportamiento en la fijación, Padilla (1973) y López et al.,(1975), han comunicado algunas observaciones generales.

El presente trabaho pretende describir el desarrollo larval y postlarval desde la veliconcha hasta la etapa de plantígrado tardío, de las principales especies de las familias: Mytilidae, Pectinidae, Pholadidae y Teredinidae, que se fijan a los colectores, incluyendo las variaciones estructurales durante la metamorfosis de mitílidos, así como observaciones sobre la fijación primaria, fenómeno descrito por diversos autores para especies del Hemisferio Norte (Bayne, 1964 ; Waine \& Wood, 1974).

\section{Materiales y Métodos}

El material estudiado se obtuvo entre los meses de Noviembre de 1977 y Mayo de 1978 , en la mitilicultura de Codihué (41 ${ }^{\circ} 46$ 'S $73^{\circ} 24 \mathrm{~W}$ ), utilizándose los siguientes métodos: a) Seis colectores de cáñamo de $16 \mathrm{~m}$, fueron suspendidos de una balsa con colectores el 11 de Noviembre de 1977 , retirándose a los $7,15,30$ y 120 días de sumersión. En cada caso se verificó individualmente el sustrato y tipo de fijación, obteniéndose de este modo, la mayor parte de los ejemplares usados en el análisis taxonómico; b) Cinco colectores confeccionados con red anchovetera ("fuelles"), provistos de dos platos ubicados a $1 \mathrm{~m}$ y $5 \mathrm{~m}$ de profundidad, se suspendieron del emparrillado de un falucho el 10 de Mayo de 1978 y retirados a los 10,30 y 58 días, fijándose inmediatamente cada plato por separado, en una solución de formol y tetraborato de sodio al $10 \%$ a $\mathrm{pH} 8$. Las larvas se obtuvieron por remoción y repetidos lavados, efectuándose luego una filtración en mallas de $75 \mu$ y $125 \mu$ de abertura; c) Con fines comparativos, se tomaron muestras quincenales de plancton, con una red Clarck - Bumpus de $76 \mu$

Para la determinación intra $\mathrm{e}$ interespecífica se tomaron como patrones, estructures presentes en juveniles con charnela definitiva, claramente identificables, que fuesen posibles de obterner al menos en dos estados consecutivos. En la nominación de estadios y estructuras, se siguieron los criterios de Rees (1950), Baune (1964) y Chanley \& Andrews (1971).

\section{Resultados}

En el colector de cáñamo sumergido durante 7 días, se contabilizaron 14 ejemplares de mitílidos, 13 de los cuales eran veliconchas cuyas alturas fluctuaron entre $300 \mu$ y $320 \mu$ y longitudes entre $320 \mu$ y $352 \mu$, con valores medios de $315 \mu$ y $330 \mu$, respectivamente. El único especimen provisto de una pequeña dissoconcha midió $385 \mu$ de largo y $396 \mu$ de alto. Diez ejemplares, incluyendo el estado post - larval, se encontraban adheridos a un alga filamentosa, Enteromorpha sp (Ulvacea, Clorophyta). En los colectores sumergidos por períodos mayores alrededor del $90 \%$ de las pedivelíferas, se encontraban fijadas a esta alga, lo que plantea la posibilidad que sea el sustrato natural, de fijación primaria; a este estado, Carriker (1961) y Bayne (1.64), le han denominado plantígrado temprano. Los restantes ejemplares, que correspondían a los de menor tamaño, se encontraban sobre el film de diatomeas que aparece tempranamente cubriendo los colectores. Ninguna larva con prodissoconcha II, se encontró fijada directamente a los filamentos de cáñamo en todos los colectores analizados. Se observaron también larvas de charnela recta, con prodissoconcha II, claramente insinuada, de tamaño superior a la prodissoconcha I, planctónica.

La pedivelífera de $A$. ater, durante la fijación primaria, (longitud: $308 \mu$ ) presenta declive anterior alargado, casi recto, margen anterior truncado y algo hendido hacia el interior, siendo esta característica más notoria en las etapas más avanzadas (Fig. 3b). Los dientes de la parte central del provinculum son bien definidos (longitud promedio: $140 \mu$; altura $8 \mu-9 \mu$, auque menores en altura que los dientes laterales (altura: $10 \mu-15 \mu$ ). (Fig. 3a.) En $M$. chilensis, tanto el declive anterior como el posterior son claramente redondeados, al igual que el margen posterior (Fig. 1b). Los dientes de la parte central del provinculum son pequeños, irregulares en disposición y tamaño, y poco definidos. La longitud del provinculum es mayor que en $A$. ater $(143 \mu$ a $160 \mu)$ aunque tanto los dientes centrales, como los de las áreas laterales son claramente inferiores en altura (Fig. 1a). El biso está formado por pocos y largos filamentos que emergen de la parte central del margen ventral, marcando una fase terminal de la metamorfosis. En este momento. los órganos internos y la boca rotan en $90^{\circ} \mathrm{y}$ el pié se desarolla notablemente. En estadios post - larvales de $A$. ater el biso se hace más poderoso, con mayor número de filamentos, diferenciando su parte basal, desplazándose paulatinamente hacia la región anterior. Un giro de $45^{\circ}$, se ha alcanzado en ejemplares de $360 \mu$ de altura. Cuando mide $520 \mu$ aproximadamente, aicanza su posición definitiva en la parte ligeramente inferior, del declive anterior.

El tamaño mínimo de fijación definitiva fue en $M$. chilensis de $720 \mu$ de altura y de alrededor de $800 \mu$ en $A$. ater. Los plant1. grados tardíos poseen una dissoconcha muy desarrolada, filamentos branquiales notorios, el ángulo antero-dorsal, sobrepasa notoriamente al ángulo póstero - dorsal y la totalidad de los dientes del provinculum son uniformes en tamaño, perdiendo nitidez, a medida que los ejemplares crecen en altura. En A. ater, la dissoconcha presenta gruesas líneas concéntricas, siendo estrecha y sin ninguna diferenciacion notoria a la altura del ángulo ántero - dorsal. El borde anterior es recto, lineal desde el ángulo antero - dorsal al inicio del margen ventral (Fig. 4a y b). M. chilensis, en cambio, presenta una dissoconcha hialina, con líneas concéntricas apretadas, no siempre apreciables, siendo amplia a la altura del ángulo ánterro - dorsal, donde existen 3 a 4 pliegues notorios. El margen anterior, es mucho menos profundo que el posterior y ampliamente redondeado (Fig. 2a y b). Los pliegues se hacen claramente visibles, cuando el plantígrado tiene una altura de $500 \mu$, aproximadamente.

El tamaño en la fijación definitiva y la eficiencia del sustrato colector, fue muy variable, debido a que se encontraron plantígrados de hasta $1,3 \mathrm{~mm}$ de algura, flotando a la deriva, lo que determina amplios rangos de talla, entre las larvas y post - larvas que colonizan los colectores (Tabla I). Para M. chilensis, en colectores de 10 días, sólo se encontraron veliconchas, en tanto a lós 30 días, el $37,50 \%$ de los especímenes midió entre $331 \mu$ y $720 \mu$ y el $45.83 \%$ entre $721 \mu$ y $1.000 \mu$; a los 58 días, el $49,50 \%$ midió entre $1.000 \mu$ y $1.500 \mu$. En $A$. ater, a los 30 días, existía igual número de especímenes que tenían alturas entre $331 \mu$ y $720 \mu$ y 721 y $1.000 \mu$. A los 58 días el $47,89 \%$, midió entre $1.000 \mu$ y $1.500 \mu$. 
Tabla I - Número y rangos de talla, de larvas y post - larvas de mitílidos en colectores artificiales.

$\begin{array}{clrlr}\text { Plato } & \mathbf{t} & \mathbf{n} & \text { Rango altura }(\mu) \\ & 1 & 2 & 310-374 \\ \text { S } & 2 & 5 & 407-946 \\ & 3 & 572 & 396-15.200\end{array}$

M. chilensis

$\begin{array}{rrrlr} & 1 & 30 & 275-594 \\ \mathrm{I} & 2 & 24 & 528-3.100 \\ & 3 & 223 & 276-7.400 \\ & & & \\ & & & 3 & 320-484 \\ & & 1 & 1 & 341 \\ & 2 & 3 & 250 & 330-12.450\end{array}$

A. ater

$\begin{array}{rrrr} & 1 & 17 & 297-363 \\ 1 & 2 & 25 & 616-5.000 \\ & 3 & 119 & 275-10.800\end{array}$

(1): 10 días; (2): 30 días; (3): 58 días; $S$ : superior; 1 : inferior.

Tanto sobre los colectores de cáñamo como de red anchovetera, se encontraron larvas y post - larvas de las familias: Teredinidae, Pholadidae y Pectinidae. La primera representada en Chile por una sola especie: Bankia martensi Stempell, 1898. El tamaño medio de las veliconchas recogidas sobre el sustrato fue: altura: $209 \mu$; longitud: $210 \mu$, aunque en el placton existían ejemplares de mayor tamaño (altura: $230 \mu-260 \mu$; longitud: $209 \mu-266 \mu$. Este estadio presenta en cada.valva dos dientes rectangulares (alto
$16 \mu$; largo: $13 \mu-15 \mu$ ) y dos cavidades. El provinculum alcanza una longitud total que fluctuó entre $46,5 \mu$ y $49 \mu$ (Fig. 7). Alrededor de la concha una banda escura, nítida y alta. La pedivelífera tiene una longitud media de $286 \mu$ y un alto de $308 \mu$; no observándose variaciones estructurales del provinculum respecto al estadio precedente.

Las veliconchas de Pholadidae, presumiblemente Pholas chiloensis (Molina, 1782), detectadas tanto en el plancton como sobre el film de diatomeas de los coléctores tienen una longitud de $170 \mu-198 \mu$ y altura: $176 \mu-181 \mu$. El provinculum presenta en la valva derecha un diente cuadrangular (longitud: $20 u-26 \mu$ ) $y$ uno de menor tamaño, teniendo en total una longitud de $52 \mu-$ $58 \mu$ (Fig. 5a y b). Los declives anterior y posterior son más rectos que en Bankia martensi y la banda oscura que rodea la concha es menos aparente. El estadio post - larval, con concha dissoconcha presenta tres crestas en el margen ventral.

De la familia Pectinidae, se encontraron juveniles de Chlamys patriae Doello Jurado, 1918 y Ciclopecten sp. En el plancton y sobre la superficie de los colectores, sólo se detectó la presencia de veliconchas de la primera especie. Longitud: $197 \mu-204 \mu$; altura: $195 \mu-210 . \mu$. Longitud del provinculum: $78 \mu-80 \mu$. El ligamento es alargado: $25 \mu-26 \mu$ de longitud, bajo el área central edéntula del provinculum: seis dientes en el lado anterior y cinco dientes en el lado posterior (Fig. 6). La metamorfosis se completa antes de que la larva alcance una longitud de $213 \mu$ y una altura de $218 \mu$. A las post - larvas no les fue observado biso, presentando valvas desiguales, la derecha es de menor tamaño y casi plana con aurículas a ambos lados, pero el anterior con una depresión pronunciada. La dissoconcha con estrías concéntricas y solo capa prismática hasta un tamaño incluso superior a $1 \mathrm{~mm}$. La valva izquierda presenta en su dissoconcha ornamentación radial, inmediatamente de anarecida ésta. Post - larvas de $410 \mu$ de altura tienen un provinculum de $84 \mu$ de largo, con ligamento alargado y dientes rectangulares, que se reducen paulatinamente hasta casi desaparecer a un tamaño de $1 \mathrm{~mm}$.

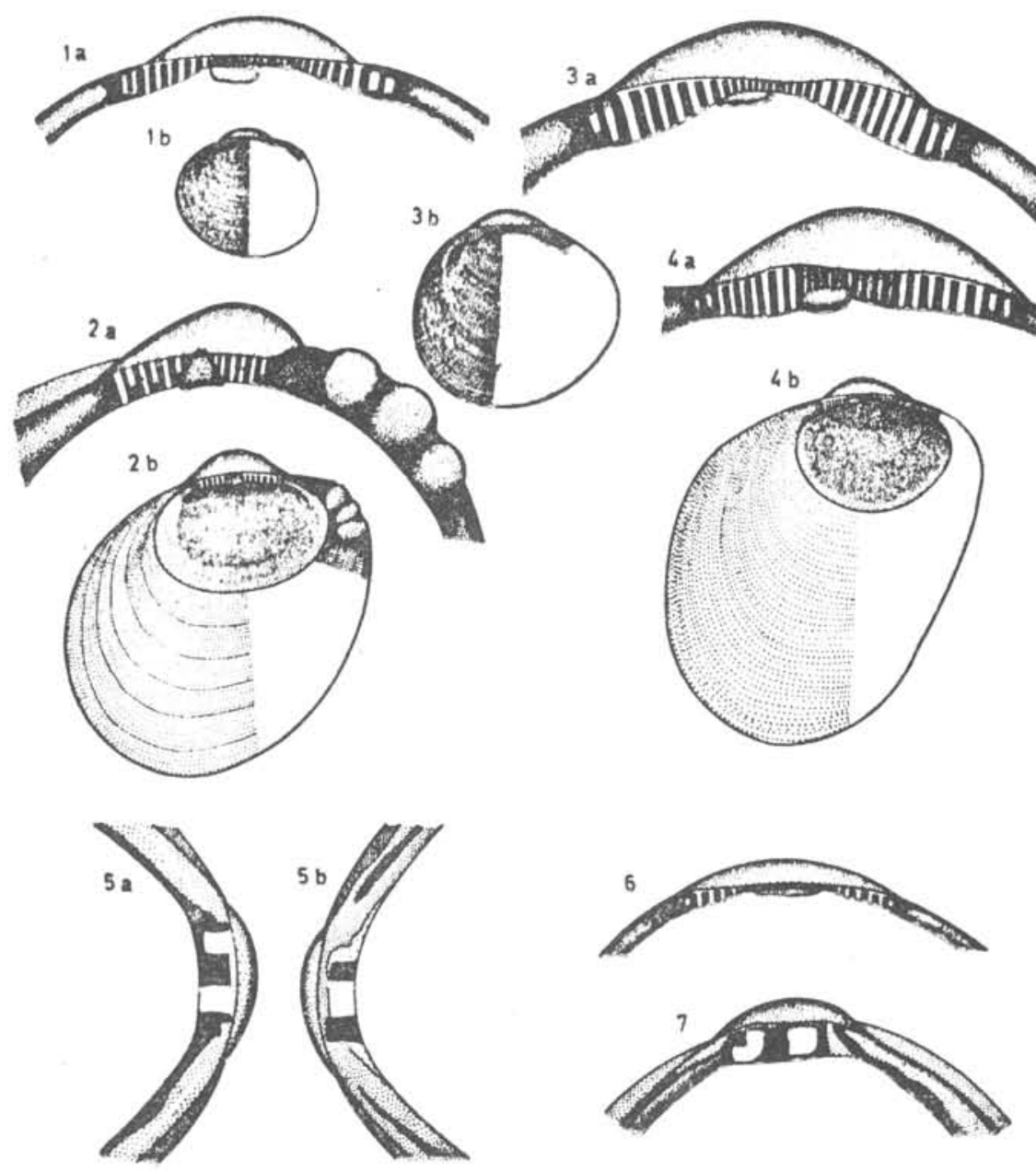

Fig. 1a. Provinculum de veliconcha de Mytilus chilensis, 330 x; Fig. 1b. Veliconcha de $M$. chilensis, $73 \mathrm{x}$; Fig. 2a. Pronvinculum de plantígraro tardío de $M$. chilensis $200 \mathrm{x}$ Fig. 2 b. Plantigrado tardío de $M$. chilensis 84 x; Fig. 3a. Provinculum de veliconcha de A ulacomys ater $464 \mathrm{x}$; Fig. 3b. Veliconcha de $A$. ater $106 \mathrm{x}$; Fig. 4a. Provinculum de plantígrado tardío de $A$ ater $380 \times$; Fig. 4 b. Plantígrado tardío de $A$ ater $94 \times ;$ Fig. $5 a$ Provinculum valva zauierda de veliconcha de Pholos chiloensis $340 \mathrm{x}$; F is. $5 \mathrm{~b}$. Provincilum valva derecha de veliconcha de $P$. chiloensis 340 x; Fig, 6 . Prrvinculum de veliconcha de Chlamys patrige $460 \mathrm{x}$; Fig 7. Provinculum de veliconcha de Bankia martensi $34 \mathrm{C} \mathrm{x}$. 


\section{Discussion y Conclusiones}

El fenómeno de fijación primaria sobre un alga filamentosa y ulterior desprendimiento, son factores importantes en la colonización de sustratos, por larvas de mitílidos. El hecho que las larvas y post - larvas tempranas muestren una mayor presencia a $5 \mathrm{~m}$ que $1 \mathrm{~m}$, en tanto los plantígrados se fijan preferentemente cerca de la superficie, supone cambios en la conducta de fijación primaria y definitiva. De igual modo el predominio de estadios larvales y post larvales de $M$. chilensis sobre $A$. ater que se invierte en colectores con juveniles de más de $3 \mathrm{~mm}$ de altura, en los que $A$. ater llega a constituir el $70 \%$ de los ejemplares, sugiere que los factores que determinan una mayor dispersión en el periodo anterior a la fijación primaria pueden operar de modo diferente en ambas especies.

Facotres como temperatura y disponibilidad de sustrato hacen variar considerablemente el período de metamorfosis (Bayne, $1965)$, no obstante la talla en que hay pié funcional y filamentos bisales ventrales, en los mitílidos estudiados nunca excedió los $300 \mu$ de altura. Chanley \& Andrews (1971), dan un rango de $215 \mu$ a $305 \mu$ para $M$. edulis y Zakhvatkina (1959) de $261 \mu$ a $348 \mu$ para $M$. galloprovincialis.

Los estados suelen presentar, en cambio, amplias variaciones de tamaño, lo que impide definir en términos morfométricos, larvas o post - larvas tanto intra como interespecificamente. No obstante la altura y longitud del provinculum, así como la estructura de los dientes, constituyen elementos importantes para diferenciar $M$. chilensis de $A$. ater, junto a disposición de márgenes, líneas de crecimiento, etc.

\section{Referencias bibliográficas}

BAYNE, B. 1964. Primary and secondary settlement in Mytilus edulis L. J. Animal Ecol.. 33:513-23.

BAYNE, B. 1965. Growth and the delay of metamorphosis of the larvae of Mvrilus edulis L. Ophelia. 2(1): 1-47

CARRIKER, M. 1961. Interrelation of funcional morphology. henavior and autoecologv in eachy sages or tor bivalve Mercenaria mercenaria. J.Elisha Mitch. Sci. Soc., 77:168-242.

CHANLEY, P. \& ANDREWS, J.1971. Aids for identification of hivalve larvae of Virginia. Malacología 11:45-119.

CIFUENTES, A. 1975. Estudio sobre la biologia y el cultivo de Mytilus chilensis, Caleta Leandro, Bahía de Concepción - Chile. Universidad de Concepción. Tesis, $124 \mathrm{D}$.

LOPEZ, M.; ROLLERI, J.; ARACENA, O. \& LOZADA, E., 1975. Captación y crecimiento de Mytilus chilensis en Putemun, Estero de Castro (Mollusca, Bivalvia, Mytilidae). Bol. Soc. Biol Concepción 40.87-101.

LOZADA, E. 1968. Contribución al estudio de la cholga Aulaco. mya ater en Putemun. Biol. Pesq.. 3: 3-39

PADILLA, M. 1973. Observaciones biológicas relacionadas con el cultivo de Mytilus edulis chilensis en Aysen. Publnes Inst. Fom. pesn. Santiago 54:1-2'

REES, C. 1950. The identification and classification of lamellibranch larvae. Hull Bull. Mar. Ecol., 3 (19): 7-104.

SOLIS, I. \& LOZADA, E. 1971 - Algunos aspectos biológicos de la cholga de Magallanes Aulacomys ater. Biol. pesq.
$5 \cdot 109-144$.

SOLIS, I., SẢNCHEZ, P. \& NAVARRETE, S. 1976 - Identificación y descripción de larvas de moluscos bivalvos en el plancton del Estero Castro. Bol. Soc. Biol. Concención, 50: 183-95.

TOMICIC, J. 1966. Contribución al estudio de la cholga, Aulaco. mya ater (Molina), en la Bahía de Mejillones. Memoria. Universidad de Chile. A ntofagasta.: 1-66

WALNE, R. \& WOOD, R. 1974. A review of some of the shellfish research undertaken at the fisheries laboratories in 1973. Shellfish inf Leaft. Minist. Agric. Fish. Food U. K.. 33:1-46.

YANEZ, R. 1974. El cultivo experimental de choros y choritos en Putemún y Talcán, Chiloé. Bol. Soc. Biol. Concepción. $48: 315-30$

ZAKHVATKINA, K. 1959. Larvae of bivalve mollusks of the Sevastopol region of the Black Sea. Akad. Nank. SSSR. Trudy Sevastopol's skoi Biologicheskoi Stantii. 11: 108-52. 